\title{
Random vs Structured Pilot Assignment in Cell-Free Massive MIMO Wireless Networks
}

\author{
Masoud Attarifar \\ University of Tehran \\ Student Member, IEEE \\ Email: m.attarifar@ut.ac.ir
}

\author{
Aliazam Abbasfar \\ University of Tehran \\ Senior Member, IEEE \\ Email: abbasfar@ut.ac.ir
}

\author{
Angel Lozano \\ Univ. Pompeu Fabra (UPF) \\ Fellow, IEEE \\ Email: angel.lozano@upf.edu
}

\begin{abstract}
This paper addresses the performance improvement that structured pilot assignment policies can bring about, relative to a random pilot assignment, in cell-free massive MIMO wireless networks. It is shown that structured policies can deliver a multiple-fold reduction in the pilot overhead required to keep pilot contamination at some acceptably low level. While the implementation of the structured policies considered in the paper might require some degree of centralized control, their performance also captures what distributed nonrandom pilot assignment schemes (e.g., greedy or collision-detecting algorithms) can hope to approach.
\end{abstract}

\section{INTRODUCTION}

Cell-free massive MIMO has been proposed as a deconstruction of cellular massive MIMO whereby the many antennas collocated at the cell sites are scattered over the network and the associations between users and cells are released. The result is a dense infrastructure of access points (APs), each featuring one or a few antennas, with every user potentially served from every AP [1]-[3]. Alternatively, cell-free massive MIMO can be seen as a form of network MIMO or cloud radio access network (C-RAN) [4]-[6] where the number of antennas is substantially larger than the number of users on any given time-frequency signaling resource.

By retaining the massive MIMO features of time-division duplexing (TDD) and excess antennas, cell-free networks inherit several virtues of cellular massive MIMO.

- A pilot overhead that scales with the number of users, rather than with the (larger) number of antennas.

- The possibility of a highly distributed implementation, whereby each AP autonomously gathers channel estimates and computes its receive beamforming and transmit conjugate beamforming coefficients, and allocates its power. Then, rather than C-RAN, which is fully centralized, this implementation could be regarded as a form of fog-RAN.

Conversely, and at the expense of extensive backhaul, the cellfree flavour of massive MIMO provides multiple advantages over its collocated cellular brethren.

- Large-scale diversity because of the distinct path loss and shadowing from every AP; this enables a more uniform degree of service.

- Proximity to the users, which increases the received signal strength.
Because the fading coherence is finite, the number of orthogonal pilot dimensions is also finite and it is impossible to maintain strict pilot orthogonality over a large network. Eventually, the system runs out of pilots and has to reuse them. This causes interference during the pilot-assisted channel estimation and, to distinguish its effects from those of regular interference during the data transmission, the term pilot contamination has conveniently been coined. In cellular massive MIMO, such contamination has been identified as an impairment that may potentially limit the performance [7]. Nonetheless, by introducing appropriate pilot reuse patterns ensuring a minimum distance among cells sharing the same pilots, the effect of the contamination can be kept at bay in cellular networks.

Cell-free networks, in contrast, do not readily lend themselves to the application of pilot reuse patterns. The most straightforward approach to assign pilots in these networks is randomly and, because of simplicity and amenability to distributed implementations, this is the almost unanimous approach in the cell-free massive MIMO literature. At the same time, and short of outright malicious schemes, a random assignment is also the worst case in terms of pilot contamination as there are no guarantees concerning the minimum distance among copilot users.

The aim of this paper is to establish the advantage over a random assignment of structured pilot assignment policies seeking to maximize the minimum distance among copilot users. While implementing policies of this nature might require some degree of centralized control, their performance captures what refined but distributed nonrandom algorithms (e.g., the greedy variety proposed in [1] or the collision-detecting approaches put forth in [8], [9]) can aspire to.

With this goal in mind, we conservatively set the features of our models (network load or AP-to-user ratio, noise floor, pathloss exponent) to favor contamination, so as to provide a worst-case diagnose. Then, at the end of the paper, we discuss how these and other aspects may affect our findings.

While explicitly modeling the reverse-link pilot transmission and ensuing channel estimation is crucial to our study, for the forward link we consider data transmission unassisted by pilots. This minimizes the analytical clutter, yielding performance metrics that lower-bound what could be achieved with further precoded pilots [10]. 
As in [1], we consider single-antenna APs, which, for a given density of antennas per unit area, represents the most distributed kind of cell-free network and thus the smallest inter-AP distance. Intermediate cases with multiantenna APs exhibiting a somewhat higher spacing are also interesting, and commented at the end.

\section{NETWORK AND CHANNEL MODELS}

The networks under consideration feature $N$ APs and $K$ users located over an area, either indoors or outdoors. All units feature a single antenna, omnidirectional in azimuth.

Every AP can communicate with every user on each timefrequency signaling resource. Thanks to the TDD, and with perfect calibration of the transmit-receive chains assumed [11], the forward and reverse channels are reciprocal. A share of the resource units are reserved for pilot transmissions from the users, based on which the channels are estimated by the APs. The remaining resource units are available for payload data transmission; these can be apportioned between the forward and reverse directions as desired.

\section{A. Large-scale Modeling}

The APs and users are randomly placed over the network area, such that their locations conform to respective (mutually independent) binomial point processes. When the network is large, these binomial point processes can be viewed as Poisson point processes.

The operating bandwidth is in the microwave range. The signals are subject to pathloss with exponent $\eta$ as well as lognormal shadowing, and the combination of both phenomena gives rise to a local-average channel gain $G_{n, k}$ between the $n$th AP the $k$ th user.

The forward- and reverse-link local-average SNRs equal $\mathrm{SNR}_{n, k}=G_{n, k} P / \sigma^{2}$ and $\mathrm{SNR}^{\mathrm{r}}=G_{n, k} P^{\mathrm{r}} / \sigma^{2}$ where $P$ and $P^{r}$ are the maximum transmit powers at APs and users, respectively, measured at $1 \mathrm{~m}$ from the corresponding source so that no scaling constants are needed; the power constraints are applied in an average sense, as corresponds to ergodic wideband channels where power fluctuations even out in the frequency domain. In turn, $\sigma^{2}$ is the noise power within the signal bandwidth.

Defining $\rho=P / P^{r}$, we can relate the local-average SNRs in both directions via

$$
\mathrm{SNR}_{n, k}^{\mathrm{r}}=\frac{\mathrm{SNR}_{n, k}}{\rho} .
$$

These local-average SNRs, and the large-scale parameters in general, are stable and known for each network snapshot.

\section{B. Small-scale Modeling}

Besides a large-scale gain $G_{n, k}$, the reverse-link channel between the $k$ th user and the $n$th AP features small-scale fading component $h_{n, k} \sim \mathcal{N}_{\mathbb{C}}(0,1)$, independent across users and APs. Under reciprocity, the forward-link channel between the $n$th AP and the $k$ th user is $h_{n, k}^{*}$. The fading exhibits coherences $T_{\mathrm{c}}$ and $B_{\mathrm{c}}$ in time and frequency, respectively. These coherences can be visualized as tiles of size $T_{\mathrm{c}} \times B_{\mathrm{c}}$ in the time-frequency plane, especially since such simple blockwise structure has been shown to be equivalent to smooth autocorrelation functions as far as the LMMSE estimation of the fading is concerned [12], [13]. The number of resource units per coherence tile equals $N_{\mathrm{c}}=B_{\mathrm{c}} T_{\mathrm{c}}$.

\section{Simulation Environment}

To generate performance distributions over many network snapshots, we resort to a wrapped-around (i.e., without boundaries) circular universe. Under the assumption that the AP positions are agnostic to the radio propagation, shadow fading has been shown to render the network approximately Poissonlike from the vantage of any user [14]. This approximation sharpens as the shadowing variance grows, being highly precise for values of interest [14], [15]. Capitalizing on this result, in our simulator the AP positions are drawn uniformly, avoiding the need for explicit modeling of the shadowing as it is then already implicitly captured by the network geometry. Likewise, the user positions are drawn uniformly.

Every result is generated from 1000 network snapshots with 5000 APs each, ensuring a $95 \%$ confidence interval of $0.3 \%$ in the results. Thanks to the wrap-around, all APs contribute equally to the statistics.

In terms of the noise floor, two distinct cases are considered.

- $P / \sigma^{2} \rightarrow \infty$, which corresponds to a pure interferencelimited network.

- $P / \sigma^{2}=45 \mathrm{~dB}$, which could be a typical value for very dense deployments. For instance, this could amount to $100 \mathrm{~mW}$ of radiated power over $100 \mathrm{MHz}$ of bandwidth, yielding an SNR of $30 \mathrm{~dB}$ at $10-20 \mathrm{~m}$ from the transmitter under reasonable values for the pathloss exponent and for the intercept at $1 \mathrm{~m}$.

\section{REVERSE-LINK CHANNEL ESTIMATION}

Let $N_{\mathrm{p}}<N_{\mathrm{c}}$ denote the number of resource units that are reserved for pilot transmissions and let $\mathcal{P}_{k}$ denote the set of users (including user $k$ ) that share the same pilot as user $k$. The simultaneous transmission from the users in this set of a pilot of power $P^{r}$ is observed at the $n$th access point as

$$
y_{n}=\sum_{\mathrm{k} \in \mathcal{P}_{k}} \sqrt{G_{n, \mathrm{k}}} h_{n, \mathrm{k}} \sqrt{P^{\mathrm{r}}}+v_{n}
$$

where $v_{n} \sim \mathcal{N}_{\mathbb{C}}\left(0, \sigma^{2}\right)$. From this observation, the LMMSE estimate of $h_{n, k}$ is

$$
\hat{h}_{n, k}=\frac{\mathrm{SNR}_{n, k}^{\mathrm{r}}}{1+\sum_{\mathrm{k} \in \mathcal{P}_{k}} \mathrm{SNR}_{n, \mathrm{k}}^{\mathrm{r}}}(h_{n, k}+\underbrace{\sum_{\substack{\mathrm{k} \neq k \\ \mathrm{k} \in \mathcal{P}_{k}}} \sqrt{\frac{\mathrm{SNR}_{n, \mathrm{k}}^{\mathrm{r}}}{\mathrm{SNR}_{n, k}^{\mathrm{r}}}} h_{n, \mathrm{k}}}_{\text {Pilot contamination }})+e_{n, k}
$$

where $e_{n, k} \sim \mathcal{N}_{\mathbb{C}}\left(0, \sigma_{e}^{2}\right)$ with

$$
\sigma_{e}^{2}=\frac{\operatorname{SNR}_{n, k}^{\mathrm{r}}}{\left(1+\sum_{\mathrm{k} \in \mathcal{P}_{k}} \operatorname{SNR}_{n, \mathrm{k}}^{\mathrm{r}}\right)^{2}} .
$$

The channel estimate's power equals

$$
\mathbb{E}\left[\left|\hat{h}_{n, k}\right|^{2}\right]=\frac{\mathrm{SNR}_{n, k}^{\mathrm{r}}}{1+\sum_{\mathrm{k} \in \mathcal{P}_{k}} \mathrm{SNR}_{n, \mathrm{k}}^{\mathrm{r}}} .
$$




\section{FORWARD-LINK DATA COMMUNICATION}

\section{A. SINR}

With conjugate beamforming based on channel estimates, the precoder applied by the $n$th AP to transmit to user $k$ is proportional to $\hat{h}_{n, k}$. With that, the $n$th AP generates the signal

$$
x_{n}=\sum_{\mathrm{k}=1}^{K} \sqrt{\frac{p_{n, \mathrm{k}} P}{\mathbb{E}\left[\left|\hat{h}_{n, \mathrm{k}}\right|^{2}\right]}} \hat{h}_{n, \mathrm{k}} s_{\mathrm{k}}
$$

where $s_{\mathrm{k}} \sim \mathcal{N}_{\mathbb{C}}(0,1)$ is the coded symbol intended for user k, the normalization by $\mathbb{E}\left[\left|\hat{h}_{n, \mathrm{k}}\right|^{2}\right]$ enforces the average power constraint, and the share of power that the $n$th AP devotes to user $\mathrm{k}$ is represented by $p_{n, \mathrm{k}}$. We let $\sum_{\mathrm{k}=1}^{K} p_{n, \mathrm{k}}=1$, meaning that each AP radiates all its available power.

The observation at the $k$ th user is

$$
y_{k}=\sum_{n=1}^{N} \sqrt{G_{n, k}} h_{n, k}^{*} x_{n}+v_{k}
$$

where $v_{k} \sim \mathcal{N}_{\mathbb{C}}\left(0, \sigma^{2}\right)$.

Without the benefit of forward pilots, the users cannot estimate their precoded fading channels and must detect their intended signals relying only on large-scale quantities. Specifically, users can exploit the channel hardening that arises for $N / K \rightarrow \infty$, i.e, when many spatial dimensions are folded over at each user [1]. Then, the precoded channels converge to their expected (over the small-scale fading) values, which can be computed on the basis of only large-scale quantities. From the vantage of such a detector, the signal recovered by user $k$ is the projection of its observation on

$$
\mathbb{E}\left[h_{n, k}^{*} \hat{h}_{n, k}\right]=\frac{\mathrm{SNR}_{n, k}^{r}}{1+\sum_{\mathrm{k} \in \mathcal{P}_{k}} \mathrm{SNR}_{n, \mathrm{k}}^{r}}
$$

where we have invoked (3). In turn, the projection on $h_{n, k}^{*} \hat{h}_{n, k}-\mathbb{E}\left[h_{n, k}^{*} \hat{h}_{n, k}\right]$ is self-interference. To better appreciate this, we can expand the combination of (4), (5), (6) and (7) into

$$
\begin{aligned}
y_{k}= & \underbrace{\sum_{n=1}^{N} \sqrt{\frac{\mathrm{SNR}_{n, k}^{r}}{1+\sum_{\mathrm{k} \in \mathcal{P}_{k}} \mathrm{SNR}_{n, \mathrm{k}}^{r}}} \sqrt{G_{n, k} p_{n, k} P} s_{k}}_{S_{k}: \text { Desired Signal }} \\
& +\underbrace{\sum_{n=1}^{N}\left(\frac{h_{n, k}^{*} \hat{h}_{n, k}}{\sqrt{\mathbb{E}\left[\left.\hat{h}_{n, k}\right|^{2}\right]}}-\sqrt{\mathbb{E}\left[\left|\hat{h}_{n, k}\right|^{2}\right]}\right) \sqrt{G_{n, k} p_{n, k} P} s_{k}}_{I_{k}: \text { Multiuser Interference }} \\
& +\underbrace{\sum_{n=1}^{N} \sqrt{G_{n, k} P} \sum_{\mathrm{k} \neq k} \frac{h_{n, k}^{*} \hat{h}_{n, \mathrm{k}}}{\sqrt{\mathbb{E}\left[\left|\hat{h}_{n, \mathrm{k}}\right|^{2}\right]}} \sqrt{p_{n, \mathrm{k}}} s_{\mathrm{k}}}_{\mathrm{E} \text { Self-Interference }}+v_{k}
\end{aligned}
$$

and express the SINR at user $k$ as

$$
\begin{aligned}
\operatorname{SINR}_{k} & =\frac{\mathbb{E}\left[\left|S_{k}\right|^{2}\right]}{\sigma^{2}+\mathbb{E}\left[\left|E_{k}\right|^{2}\right]+\mathbb{E}\left[\left|I_{k}\right|^{2}\right]} \\
& =\frac{\frac{1}{\sigma^{2}} \mathbb{E}\left[\left|S_{k}\right|^{2}\right]}{1+\frac{1}{\sigma^{2}} \mathbb{E}\left[\left|E_{k}\right|^{2}\right]+\frac{1}{\sigma^{2}} \mathbb{E}\left[\left|I_{k}\right|^{2}\right]}
\end{aligned}
$$

where

$$
\frac{1}{\sigma^{2}} \mathbb{E}\left[\left|S_{k}\right|^{2}\right]=\left(\sum_{n=1}^{N} \sqrt{\frac{p_{n, k} \mathrm{SNR}_{n, k}^{\mathrm{r}} \mathrm{SNR}_{n, k}}{1+\sum_{\mathrm{k} \in \mathcal{P}_{k}} \mathrm{SNR}_{n, \mathrm{k}}^{\mathrm{r}}}}\right)^{2} .
$$

With a bit of algebra $\frac{1}{\sigma^{2}} \mathbb{E}\left[\left|E_{k}\right|^{2}\right]$ and $\frac{1}{\sigma^{2}} \mathbb{E}\left[\left|I_{k}\right|^{2}\right]$ can also be expressed and the SINR can be given in the explicit and remarkably compact form [1]

$$
\operatorname{SINR}_{k}=\frac{\left(\sum_{n=1}^{N} \sqrt{\frac{p_{n, k} \mathrm{SNR}_{n, k}^{2}}{\rho+\sum_{\mathrm{k} \in \mathcal{P}_{k}} \mathrm{SNR}_{n, \mathrm{k}}}}\right)^{2}}{1+\sum_{n=1}^{N} \mathrm{SNR}_{n, k}+\sum_{\substack{\mathrm{k} \neq k \\ \mathrm{k} \in \mathcal{P}_{k}}}\left(\sum_{n=1}^{N} \sqrt{\frac{p_{n, \mathrm{k}} \mathrm{SNR}_{n, k}^{2}}{\rho+\sum_{\mathrm{k}^{\prime} \in \mathcal{P}_{\mathrm{k}}} \mathrm{SNR}_{n, \mathrm{k}^{\prime}}}}\right)^{2}}
$$

which is expressed as function of only the forward-link SNRs thanks to the relationship $\mathrm{SNR}_{n, k}^{\mathrm{r}}=\mathrm{SNR}_{n, k} / \rho$, and where we have used $\sum_{\mathrm{k}=1}^{K} p_{n, \mathrm{k}}=1$.

As $N_{\mathrm{p}} \rightarrow \infty$, the set $\mathcal{P}_{k}$ comes to contain only user $k$ and (12) converges to

$$
\operatorname{SINR}_{k}^{\infty}=\frac{\left(\sum_{n=1}^{N} \sqrt{\frac{p_{n, k} \mathrm{SNR}_{n, k}^{2}}{\rho+\mathrm{SNR}_{n, k}}}\right)^{2}}{1+\sum_{n=1}^{N} \mathrm{SNR}_{n, k}}
$$

with the pilot contamination rendered negligible.

\section{B. Spectral Efficiency}

With a decoder treating $E_{k}$ and $I_{k}$ as additional noise, user $k$ would achieve, over the signaling resources apportioned to the forward link, a gross spectral efficiency of [16]

$$
C_{k}=\log _{2}\left(1+\mathrm{SINR}_{k}\right)
$$

to which an overhead factor $\left(N_{\mathrm{c}}-N_{\mathrm{p}}\right) / N_{\mathrm{c}}$ would have to be applied in order to obtain the net spectral efficiency. We hasten to recall that (14), which formally corresponds to a hardening-based receiver, can also be interpreted as a lower bound for implementations featuring precoded forward pilots, as well as for alternative transmission schemes not requiring forward pilots, e.g., those based on non-Gaussian codewords and noncoherent detection [17], [18].

For $N_{\mathrm{p}} \rightarrow \infty$, the spectral efficiency converges to $C_{k}^{\infty}=$ $\log _{2}\left(1+\operatorname{SINR}_{k}^{\infty}\right)$.

The averages of $C_{k}$ and $C_{k}^{\infty}$ over all users in the network are respectively denoted by $\bar{C}$ and $\bar{C}^{\infty}$.

\section{Power Allocation}

Although the APs are privy to small-scale channel estimates, in the face of hardening it would hardly be advantageous to allocate power based on those estimates rather than directly on large-scale quantities. Within the latter class of power allocations, the natural complement to conjugate beamforming is maximal-ratio transmission, i.e., proportionality between the 


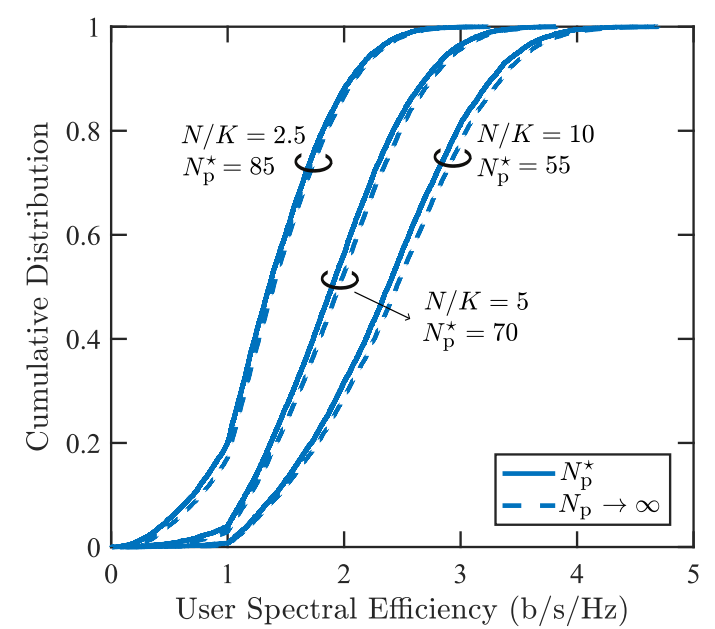

(a) $P / \sigma^{2} \rightarrow \infty$

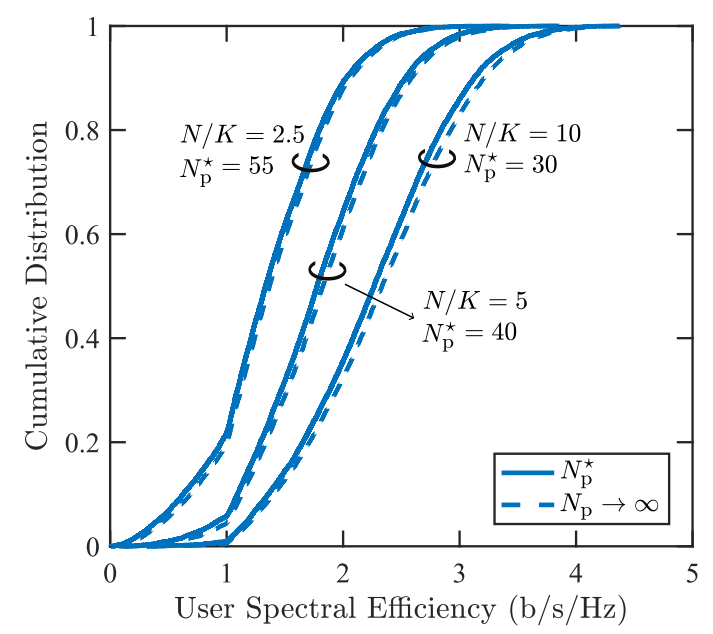

(b) $P / \sigma^{2}=45 \mathrm{~dB}$

Fig. 1. Random pilot assignment. CDF of $C_{k}$ parameterized by $N / K$ and by the noise strength. In solid, with $N_{\mathrm{p}}^{\star}$; in dashed, for $N_{\mathrm{p}} \rightarrow \infty$.

power transmitted to each user and the average strength of the corresponding channel. This amounts to

$$
\begin{aligned}
p_{n, k} & =\frac{G_{n, k}}{\sum_{\mathrm{k}=1}^{K} G_{n, \mathrm{k}}} \\
& =\frac{\mathrm{SNR}_{n, k}}{\sum_{\mathrm{k}=1}^{K} \mathrm{SNR}_{n, \mathrm{k}}}
\end{aligned}
$$

which is considered in the sequel for the sake of specificity. This allocation can be modified to enhance fairness at the expense of sum spectral efficiency [1], and we discuss at the end of the paper how our findings may change with power allocations other than (16).

\section{Pilot Assignment}

\section{A. Random Pilot Assignment}

Suppose that each user is assigned at random one of the $N_{\mathrm{p}}$ pilots. Let us determine the smallest value of $N_{\mathrm{p}}$ such that
TABLE I

$N_{\mathrm{p}}^{\star}$ THAT CAUSES A $3 \%$ LOSS IN AVERAGE GROSS SPECTRAL EFFICIENCY RELATIVE TO $N_{\mathrm{p}} \rightarrow \infty$. RANDOM PILOT ASSIGNMENT WITH $\eta=4$.

\begin{tabular}{|c|c|c|}
\hline & $P / \sigma^{2} \rightarrow \infty$ & $P / \sigma^{2}=45 \mathrm{~dB}$ \\
\hline \hline$N / K=2.5$ & $N_{\mathrm{p}}=85$ & $N_{\mathrm{p}}=55$ \\
\hline$N / K=5$ & $N_{\mathrm{p}}=70$ & $N_{\mathrm{p}}=40$ \\
\hline$N / K=10$ & $N_{\mathrm{p}}=55$ & $N_{\mathrm{p}}=30$ \\
\hline
\end{tabular}

TABLE II

$N_{\mathrm{p}}^{\star}$ THAT CAUSES A $3 \%$ LOSS IN AVERAGE GROSS SPECTRAL EFFICIENCY RELATIVE TO $N_{\mathrm{p}} \rightarrow \infty$. RANDOM PILOT ASSIGNMENT WITH $\eta=3.5$.

\begin{tabular}{|c|c|c|}
\hline & $P / \sigma^{2} \rightarrow \infty$ & $P / \sigma^{2}=45 \mathrm{~dB}$ \\
\hline$N / K=2.5$ & $N_{\mathrm{p}}=240$ & $N_{\mathrm{p}}=120$ \\
\hline$N / K=5$ & $N_{\mathrm{p}}=185$ & $N_{\mathrm{p}}=95$ \\
\hline$N / K=10$ & $N_{\mathrm{p}}=145$ & $N_{\mathrm{p}}=75$ \\
\hline
\end{tabular}

the degradation in user spectral efficiency is "negligible". For the sake of concreteness, we set "negligible" at $3 \%$, although the same exercise could be conducted for any other threshold. For various $\eta$ and $N / K$ then, we identify the values $N_{\mathrm{p}}^{\star}$ that render $\bar{C}=0.97 \bar{C}_{\infty}$ and list those values in Tables I-II. The following can be observed.

- $N_{\mathrm{p}}^{\star}$ increases markedly as the pathloss exponent shrinks and the spatial signal containment abates.

- $N_{\mathrm{p}}^{\star}$ diminishes with growing $N / K$, as users are sparsified relative to the APs, i.e., the distance between users increases relative to the distance between APs.

- The presence of noise, which masks the pilot contamination, further reduces $N_{\mathrm{p}}^{\star}$.

To evidence that the average performance is actually representative for all users, Fig. 1 presents the spectral efficiency CDFs for the identified values of $N_{\mathrm{p}}$ and for $N_{\mathrm{p}} \rightarrow \infty$, for $\eta=4$. In all cases, the effect of pilot contamination is rather uniform across the user population.

\section{B. Clustering Pilot Assignment}

We next present a policy that seeks to create regular pilot reuse structures so as to maximize the minimum distance among copilot users. The idea is to partition the $K$ users into $N_{\mathrm{p}}$ disjoint subsets, which each such subset containing $K / N_{\mathrm{p}}$ copilot users whose minimum distance is as large as possible. For a given number of users, the solution that maximizes the minimum distance is the one where the distances among users are all equal, hence we seek to identify subsets that approach this ideal.

A possible way to identify a subset of copilot users with similar distances among the members would be to overlay onto the network a regular grid with $K / N_{\mathrm{p}}$ nodes and then declare as members of the subset those users closest to the nodes. However, this procedure would be subject to the arbitrariness of which grid is chosen and how it is positioned and oriented; moreover, this procedure would work poorly near the network edges. A suitable tool to more naturally define the subsets is the k-means clustering method, an iterative algorithm that serves to partition data points into a predefined number of 


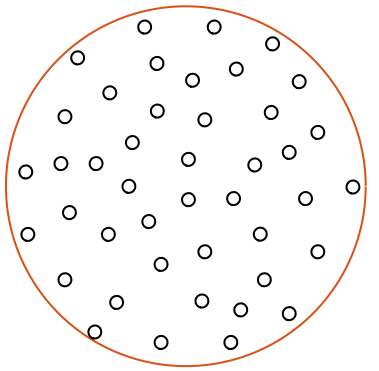

(a) (b)
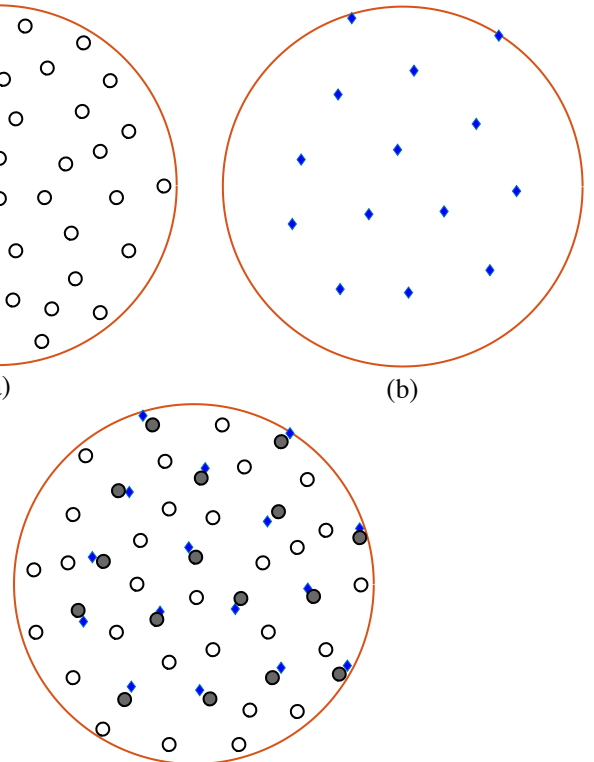

(c)

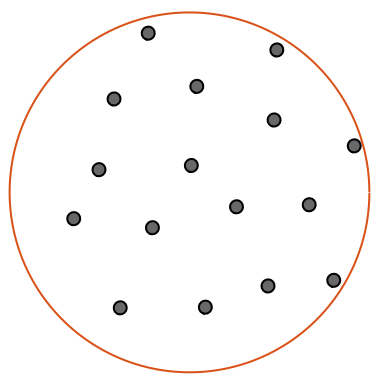

(d)

Fig. 2. Formation of copilot user subsets on a circular universe where, for clarity, the APs are not shown. (a) Population of users; (b) Centroids obtained by applying the k-means clustering method; (c) Superposition of centroids and users; (d) Subset of copilot users identified by proximity to the centroids.

clusters with each point belonging to the cluster with the nearest mean (or centroid). The algorithm operates as follows. Random points are chosen as initial centroids, and each point is assigned to its nearest centroid. Then, the centroid positions are updated by averaging over the points belonging to the respective clusters, the points are reassigned, and so on. Once the clustering has taken place, the users closest to the centroids are identified as forming a subset of copilot users. An example of this procedure is presented in Fig. 2 .

The values of $N_{\mathrm{p}}^{\star}$ that yield $\bar{C}=0.97 \bar{C}_{\infty}$ under clustering pilot assignment are summarized in Tables III-IV. In turn, Fig. 3 presents the entire CDF of gross spectral efficiency for $N_{\mathrm{p}}^{\star}$ and $N_{\mathrm{p}} \rightarrow \infty$, for $\eta=4$, confirming that the effect of pilot contamination is again rather uniform for all users.

In light of the uniformity of the degradation, meaningful conclusions can be drawn on the basis of network averages. Contrasting Tables I-II with Tables III-IV, the conclusion is that the clustering pilot assignment reduces the number of necessary pilots by a factor that ranges between 3 and 3.75.
TABLE III

$N_{\mathrm{p}}^{\star}$ THAT CAUSES A $3 \%$ LOSS IN AVERAGE GROSS SPECTRAL EFFICIENCY RElative to $N_{\mathrm{p}} \rightarrow \infty$. Clustering PILOt ASSIGNMENT With $\eta=4$.

\begin{tabular}{|c|c|c|}
\hline & $P / \sigma^{2} \rightarrow \infty$ & $P / \sigma^{2}=45 \mathrm{~dB}$ \\
\hline$N / K=2.5$ & $N_{\mathrm{p}}=25$ & $N_{\mathrm{p}}=15$ \\
\hline$N / K=5$ & $N_{\mathrm{p}}=20$ & $N_{\mathrm{p}}=12$ \\
\hline$N / K=10$ & $N_{\mathrm{p}}=17$ & $N_{\mathrm{p}}=9$ \\
\hline
\end{tabular}

TABLE IV

$N_{\mathrm{p}}^{\star}$ THAT CAUSES A $3 \%$ LOSS IN AVERAGE GROSS SPECTRAL EFFICIENCY RELATIVE To $N_{\mathrm{p}} \rightarrow \infty$. Clustering PILOT ASSIGNMENT With $\eta=3.5$.

\begin{tabular}{|c|c|c|}
\hline & $P / \sigma^{2} \rightarrow \infty$ & $P / \sigma^{2}=45 \mathrm{~dB}$ \\
\hline$N / K=2.5$ & $N_{\mathrm{p}}=64$ & $N_{\mathrm{p}}=38$ \\
\hline$N / K=5$ & $N_{\mathrm{p}}=51$ & $N_{\mathrm{p}}=30$ \\
\hline$N / K=10$ & $N_{\mathrm{p}}=42$ & $N_{\mathrm{p}}=25$ \\
\hline
\end{tabular}

\section{Summary AND Discussion}

Relative to straightforward random assignment, a structured pilot assignment scheme based on clustering can reduce by a factor of 3-3.75 the number of pilot dimensions $N_{\mathrm{p}}^{\star}$ required for the effect of contamination to be negligible (precisely, $3 \%$ in average spectral efficiency). This informs of the reduction in overhead that can be approached by nonrandom distributed algorithms or by outright centralized pilot assignments. Depending on the coherence $N_{\mathrm{c}}$, this reduction may be more or less significant. Moreover, with multiantenna rather than single-antenna APs, $N_{\mathrm{p}}^{\star}$ is expected to multiply and hence the advantage of structuring would become far more appealing.

Additional observations from our conjugate-beamforming results are the following.

- $N_{\mathrm{p}}^{\star}$ is very sensitive to the pathloss exponent, growing as $\eta$ diminishes. However, the advantage of structured pilot assignments holds steady.

- As $N / K$ shrinks, the number of users increases relatively and hence $N_{\mathrm{p}}^{\star}$ also increases. However, the increase is sublinear because there is an offsetting effect: the beams become less sharp, and thus there is additional multiuser interference masking the contamination.

- Because noise masks the contamination, the worstcase scenario in terms of pilot contamination is an interference-limited network.

To finalize the paper, let us briefly discuss the potential impact of other aspects.

- Alternative beamforming techniques. With more sophisticated beamforming schemes, say ZF, multiuser interference would decline and there would be reduced masking of the contamination. This would make structured assignments more appealing.

- Multislope pathloss. With a structured pilot allocation, this would decrease the strength of unintended copilot signals relative to the desired ones, therefore alleviating the contamination problem. This effect would be somewhat blurred with a random pilot allocation, and thus a multislope pathloss is expected to improve the relative advantage of structured allocations. 


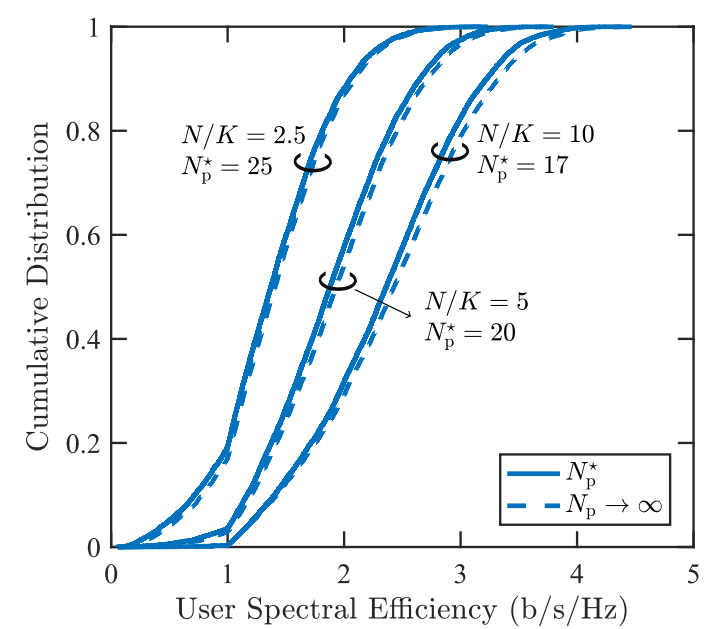

(a) $P / \sigma^{2} \rightarrow \infty$

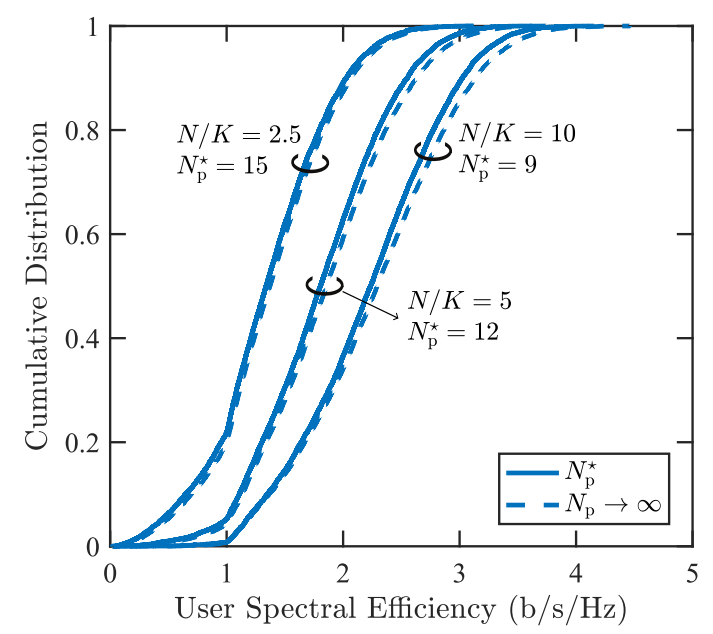

(b) $P / \sigma^{2}=45 \mathrm{~dB}$

Fig. 3. Clustering pilot assignment. CDF of $C_{k}$ parameterized by $N / K$ and by the noise strength. In solid, with $N_{\mathrm{p}}^{\star}$; in dashed, for $N_{\mathrm{p}} \rightarrow \infty$.

- Forward-link pilots. With coherent rather than hardeningbased reception, the self-interference would disappear and, with less masking, the contamination might become more pronounced. Again, this would make structured assignments more enticing.

- Power allocation. Since the impact of contamination, and the benefits of structured assignments, are rather uniform across the user population, the modification of the power allocation may not significantly alter the foregoing observations. More substantial may be the lifting of the assumption that the APs always transmit at full power.

To render these qualitative comments precise, the impact of these aspects will be quantified in follow-up work.

\section{ACKNOWLEDGMENT}

The work of A. Lozano was supported by Project TEC201566228-P (MINECO/FEDER, UE) and by the European Research Council under the H2020 Framework Programme/ERC grant agreement 694974.

\section{REFERENCES}

[1] H. Q. Ngo, A. Ashikhmin, H. Yang, E. G. Larsson, and T. L. Marzetta, "Cell-free massive MIMO versus small cells," IEEE Trans. on Wireless Communications, vol. 16, no. 3, pp. 1834-1850, 2017.

[2] E. Nayebi, A. Ashikhmin, T. L. Marzetta, H. Yang, and B. D. Rao, "Precoding and power optimization in cell-free massive MIMO systems," IEEE Trans. on Wireless Communications, vol. 16, no. 7, pp. 4445-4459, Jul. 2017.

[3] E. Nayebi, A. Ashikhmin, T. L. Marzetta, and B. D. Rao, "Performance of cell-free massive MIMO systems with MMSE and LSFD receivers," in Asilomar Conf. on Signals, Systems and Computers, Nov. 2016, pp. 203-207.

[4] M. K. Karakayali, G. J. Foschini, and R. A. Valenzuela, "Network coordination for spectrally efficient communications in cellular systems," IEEE Wireless Commun. Mag., vol. 3, no. 14, pp. 56- 61, Aug. 2006.

[5] A. Lozano, R. W. Heath, and J. G. Andrews, "Fundamental limits of cooperation," IEEE Trans. on Inform. Theory, vol. 59, no. 9, pp. 52135226, 2013.

[6] A. Checko, H. L. Christiansen, Y. Yan, L. Scolari, G. Kardaras, M. S. Berger, and L. Dittmann, "Cloud RAN for mobile networks - a technology overview," IEEE Communications Surveys \& Tutorials, vol. 17, no. 1, pp. 405-426, 2015.

[7] T. L. Marzetta, "Noncooperative cellular wireless with unlimited numbers of base station antennas," IEEE Trans. on Wireless Communications, vol. 9, no. 11, pp. 3590-3600, Nov. 2010.

[8] O. Y. Bursalioglu, C. Wang, H. Papadopoulos, and G. Caire, "RRH based massive MIMO with 'on the fly' pilot contamination control," in IEEE Int'l Conf. on Communications (ICC'16), 2016, pp. 1-7.

[9] — "A novel alternative to cloud RAN for throughput densification: Coded pilots and fast user-packet scheduling at remote radio heads," in Asilomar Conference on Signals, Systems and Computers, Nov. 2016, pp. 3-10.

[10] G. Interdonato, H. Q. Ngo, E. G. Larsson, and P. Frenger, "How much do downlink pilots improve cell-free massive MIMO?" in IEEE Global Communications Conf. (GLOBECOM'16), 2016, pp. 1-7.

[11] J. Vieira, F. Rusek, and F. Tufvesson, "Reciprocity calibration methods for massive MIMO based on antenna coupling," in IEEE Global Communications Conference (GLOBECOM'14), 2014, pp. 3708-3712.

[12] N. Jindal and A. Lozano, "A unified treatment of optimum pilot overhead in multipath fading channels," IEEE Trans. on Communications, vol. 58, no. 10 , pp. 2939-2948, Oct. 2010.

[13] A. Lozano and D. Porrat, "Non-peaky signals in wideband fading channels: achievable bit rates and optimal bandwidth," IEEE Trans. on Wireless Communications, vol. 11, no. 1, pp. 246-257, Jan. 2012.

[14] B. Błaszczyszyn, M. K. Karray, and H. P. Keeler, "Wireless networks appear Poissonian due to strong shadowing," IEEE Trans. on Wireless Communications, vol. 14, no. 8, pp. 4379-4390, Aug. 2015.

[15] G. George, R. K. Mungara, A. Lozano, and M. Haenggi, "Ergodic spectral efficiency in MIMO cellular networks," IEEE Trans. on Wireless Communications, vol. 16, no. 5, pp. 2835-2849, 2017.

[16] A. Lapidoth and S. Shamai, "Fading channels: how perfect need "perfect side information" be?" IEEE Trans. on Inform. Theory, vol. 48, no. 5, pp. 1118-1134, 2002.

[17] T. L. Marzetta and B. M. Hochwald, "Capacity of a mobile multipleantenna communication link in Rayleigh flat fading," IEEE Trans. Inform. Theory, vol. 45, no. 1, pp. 139-157, Jan. 1999.

[18] G. Caire, "On the ergodic rate lower bounds with applications to massive MIMO," arXiv preprint arXiv:1705.03577, 2017. 